Open Journal of Geriatrics

ISSN: 2639-359X

Volume 1, Issue 2, 2018, PP: 38-40

SRYPAHWA

\title{
Photo Quiz: Why did this Patient with End-Stage Renal Failure Develop Lower Extremity Ulcers?
}

Cheryl Atherley-Todd, MD, CMD, FAAFP

Associate Professor Family Medicine/Geriatrics, Dr. Kiran C. Patel College of Osteopathic Medicine, Nova Southeastern University, 3200 S University Drive, Fort Lauderdale, FL.

ca765@nova.edu

*Corresponding Author: Cheryl Atherley-Todd, MD, CMD, FAAFP Associate Professor Family Medicine/Geriatrics, Dr. Kiran C. Patel College of Osteopathic Medicine, Nova Southeastern University, 3200 S University Drive, Fort Lauderdale, FL.

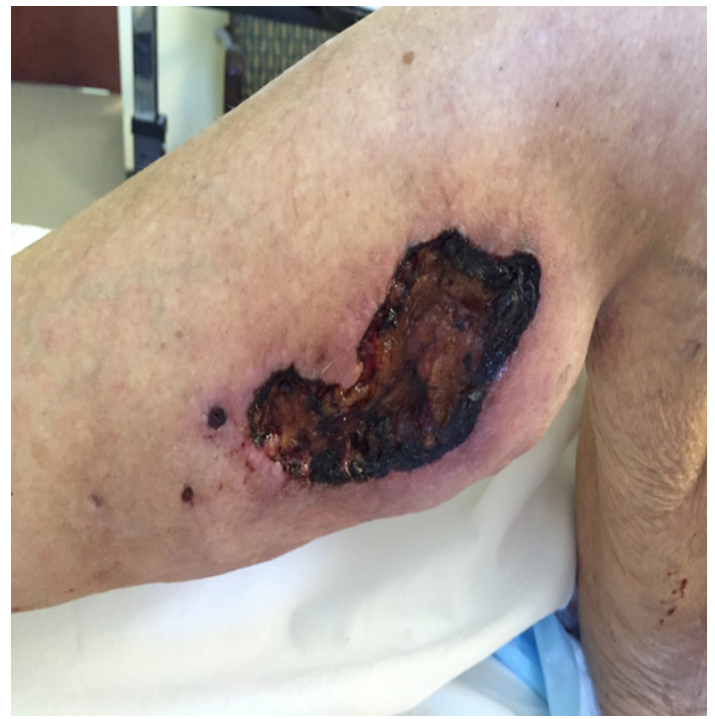

Figure 1. Ulcer on right calf

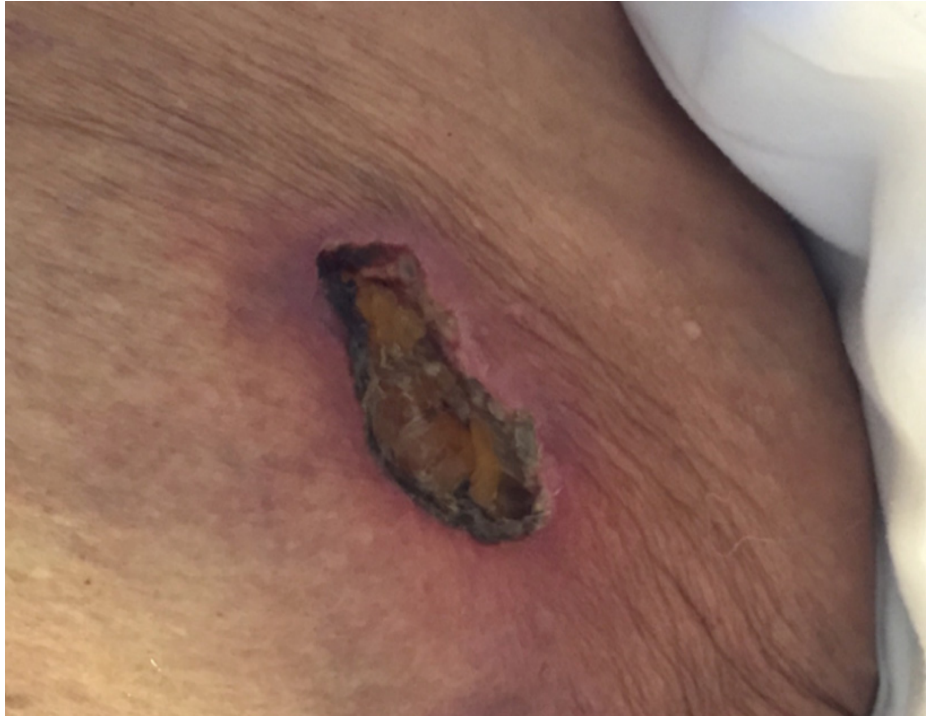

Figure 2. Necrotic ulcer on upper medial thigh

A 59 year old female developed multiple painful lesions on her lower extremities and buttocks while hospitalized for diverticulitis. She had a history of type 2 diabetes mellitus, and end-stage renal disease on hemodialysis. She had no history of peripheral vascular disease nor trauma to the affected areas.

Physical examination revealed exquisitely tender ulcers on the medial aspect of her right calf (Figure 1), lateral aspect of her upper thighs (Figure2) and on her buttocks.

\section{QUESTION}

Based on the patient's history and physical examination, which one of the following is the most likely diagnosis?
A. Tophaceous gout
B. Vibrio vulnificus infection
C. Brown recluse spider bite
D. Osteoma cutis
E. Calciphylaxis 
Photo Quiz: Why did this Patient with End-Stage Renal Failure Develop Lower Extremity Ulcers?

\section{DiscUSSION}

The answer is E: Calciphylaxis (calcific uremic arteriolopathy) is a rare disorder of systemic arterial calcification., ${ }^{1,}$ It is seen mainly in patients with chronic renal failure. The etiology is unknown but hyperparathyroidism, Vitamin D supplementation, elevated calcium and phosphorus play a role. The lesions appear suddenly and progress rapidly. They usually appear on the lower extremities and buttocks but may also occur on the breasts and hands. Patients complain of severe pain. The lesions initially look like violaceous mottling or erythematous papules, plaques or nodules. They progress to form patches of ischemic necrosis.

Treatment is mainly supportive but sodium thiosulfate intravenously at the end of each hemodialysis session along with aggressive efforts to reduce calcium $\mathrm{x}$ phosphate product has resulted in considerable improvement.

The prognosis is poor. The necrotic ulcers get infected and this can lead to sepsis and death.

Tophaceous gout is a chronic form of gout in which monosodium urate crystals are deposited in soft tissue. ${ }^{3}$ Common sites are fingers, elbows and big toe.
This is not usually painful. Tophi may be aspirated and the expressed material will show needle shaped birefringent crystals under polarized light microscopy.

Vibrio vulnificus infections are usually acquired while handling shellfish (especially oysters) or fish or after exposure of preexisting leg wounds to estuarine waters. ${ }^{4}$ Lesions are usually on the hands or legs. The diagnosis is confirmed by wound culture. The mortality rate is high due to septicemia.

Brown recluse spider bites usually occur if someone puts on clothing or footwear in which the spider sought refuge.$^{5}$ Common sites of bites are upper arms, thorax and inner thighs. Occasionally the bite site gets progressively more painful and the lesion becomes necrotic. Most lesions heal by secondary intention without scarring.

Osteoma cutis presents as hard painless nodules in the skin. ${ }^{6}$ Most commonly found in the scalp, face, digits, and subungual areas. This may have a familial association with Albright hereditary osteodystrophy. Lesions may be present from birth or they may develop secondary to inflammatory skin diseases, trauma, scars or cutaneous tumors.
Summary Table
CONDITION
Tophaceous gout
Vibrio vulnificus infection
Brown recluse spider bite
Osteoma cutis
Calciphylaxis
CHARACTERISTICS
History of gout. Tophi usually painless. Common sites: ears, fingers, elbows and big toe.
History of handling shellfish or fish or exposure to estuarine waters. Common sites: hands and legs. May have a high mortality rate.
History of spider bite. Common sites: upper arms, thorax and inner thighs. Most lesions heal without scarring.
History of lesions from birth or they may develop secondary to some insult to the skin. Usually painless. Common sites: scalp, face, digits and subungual areas.
History of chronic renal failure. Excruciatingly painful. Common sites: lower extremities and buttocks. High mortality rate. 
Photo Quiz: Why did this Patient with End-Stage Renal Failure Develop Lower Extremity Ulcers?

\section{REFERENCES}

[1] Calciphylaxis. Julia R. Nunley, MD. Medscape. Updated: Jul 21, 2014

[2] Harrison's Principles of Internal Medicine. $16^{\text {th }} \mathrm{Ed}$, McGraw Hill, Med Pub Division (2005) Ch.335

[3] Clinical manifestations and diagnosis of gout. Michael A. Becker, MD uptodate.com
[4] Vibrio vulnificus oysters: Pearls and perils. Nicholas A Daniels, MD, MPH. Clinical Infectious Diseases (2011) Vol 52, Issue 6, 788-792.

[5] Spider bite. Isbister GK. Lancet (2011) Vol 378, Issue 9808, 2039-2047.

[6] Osteoma cutis. Luke Lennox, MD. Medscape. Updated: Mar 20, 2015.

Citation: Cheryl Atherley-Todd. Photo Quiz: Why did this Patient with End-Stage Renal Failure Develop Lower Extremity Ulcers?. Open Journal of Geriatrics. 2018; 1(2): 38-40.

Copyright: (C) 2018 Cheryl Atherley-Todd. This is an open access article distributed under the Creative Commons Attribution License, which permits unrestricted use, distribution, and reproduction in any medium, provided the original work is properly cited. 